\title{
A CHARACTERIZATION OF THE VERONESE SURFACE
}

\author{
EDOARDO BALLICO
}

(Communicated by Louis J. Ratliff, Jr.)

\begin{abstract}
Here we prove a slight modification of a conjecture of BeltramettiSommese proving that the Veronese surface and a.general intersection of 3 quadrics are the only smooth surfaces of $\mathbf{C P}^{5}$ which are 2-spanned.
\end{abstract}

The aim of this short note is the proof of a slight modification of a conjecture raised in [BS, Conjecture 2.6], proving a slightly different and slightly more general result (see Theorem 1). The main tools will be an enumerative formula, a well-known theorem on projective curves and the classification given in [BS, $\S 5]$, for embedded surfaces of sectional genus at most 5 . To state the result we need to introduce a few notations. We work over the complex number field. Let $S$ be a smooth, complete surface, $L \in \operatorname{Pic}(S)$ and $W \subseteq H^{0}(S, L)$. A finite subscheme $Z$ of $S$ is called curvilinear if it is contained in a smooth curve, i.e. if for all points $P \in \operatorname{Supp}(Z), Z$ is given around $P$ by equations $x=y^{m}=0, x$ and $y$ suitable local coordinates around $P$. According to [BFS] and [BS], we say that $W k$-spans $S, k$ an integer $\geq 0$, if for all curvilinear subschemes $Z$ of $S$ with length $(Z)=k+1$, the restriction map from $W$ to $H^{0}(Z, L \mid Z)$ is surjective. $L$ is called $k$-spanned if $H^{0}(S, L) k$ spans $S$. A smooth surface $S \subset \mathbf{P}^{h}$ is called $k$-spanned if the linear system on $S$ determined by the embedding in $\mathbf{P}^{n} k$-spans $S$. There are other, perhaps more natural, definitions of $k$-spannedness (see [BFS, $\S 4]$ ), but not only is the one given here is the one used heavily in [BFS] and [BS], but also it seems the weakest one among the natural possible definitions, and so the one with which Theorem 1 is strongest. It is easy to check that if $W$ is $(k+1)$-spanned, then it is $k$-spanned. In [BFS, 0.4.1], it was noted that $W$ is 1 -spanned if and only if it embeds $S$ in a projective space (this is true even for noncomplete linear systems). In [BS, 2.4], as a corollary of a more general result, it was proved that if $L \quad k$-spans $S$ for some $k \geq 2$, then $H^{0}(S, L) \geq 6$. In [BS, 2.6], it was conjectured that if $L \quad k$-spans $S$ for some $k \geq 2$ and $H^{0}(S, L)=6$, then $S \cong \mathbf{P}^{2}$ and $L$ gives the Veronese embedding. It is easy to check that the Veronese surface in $\mathbf{P}^{5}$ is 2-spanned; this follows also from general results

Received by the editors February 22, 1988, and in revised form, July 28, 1988.

1980 Mathematics Subject Classification (1985 Revision). Primary 14J25; Secondary 14N05. 
in [BFS]: $L$ is the tensor power of two very ample line bundles. This note contains only the proof of the following theorem.

Theorem 1. Assume that $W$ 2-spans the smooth, complete surface $S$. Then $\operatorname{dim}(W) \geq 6$ and if $\operatorname{dim}(W)=6$, then either $S \cong \mathbf{P}^{2}$ and $W$ gives the Veronese embedding into $\mathbf{P}^{5}$, or $S$ is the intersection of 3 quadric hypersurfaces. In the latter case $S$ is 2-spanned if and only if it contains no line; the set of such smooth complete intersections containing at least a line forms a non-empty hypersurface in the variety of all smooth complete intersections of 3 quadric hypersurfaces in $\mathbf{P}^{5}$.

In particular note that the Veronese surface is the only smooth surface in $\mathbf{P}^{5}$ such that all smooth embedded deformations in $\mathbf{P}^{5}$ are again 2-spanned.

Note that by definition $S \subset \mathbf{P}^{n}$ is a 2-spanned surface, then there is no line $D \subset S$ and no line $R \not \subset S$ with length $(R \cap S) \geq 3$ (a "trisecant line"). Thus $\operatorname{dim}(W)>4$. Consider $S$ embedded in $\mathbf{P}^{n}$ by $W$. Then for any hyperplane $H, H \cap S$ contains no "trisecant line". For general $H, S \cap H$ is a smooth, irreducible space curve (Bertini's theorem). Set $d:=\operatorname{deg}(S)$ and let $g$ be the genus of $S \cap H$.

First assume $\operatorname{dim}(W)=5$. Fix a point $P$ of $S \cap H$; there is no "trisecant" line to $S \cap H$ passing through $P$ if and only if the image of the projection of $S \cap H$ from $P$ into $\mathrm{P}^{2}$ has degree $d-1$ and is isomorphic to $S \cap H$, hence of arithmetic genus $g$. By the genus formula for plane curves and Castelnuovo's bound for the genus of space curves ([ACGH, p. 116]), one sees immediately that for any point $P$ of $S \cap H$, there is a "trisecant line" to $S \cap H$ passing through $P$, unless $(d, g)=(3,0)$ or $(4,1)$. If $d=3$, then $S$ is a minimal degree surface in $\mathbf{P}^{4}$, hence a scroll, hence it contains many lines, contradiction. Assume $(d, g)=(4,1)$. Since $S \cap H$ is linearly normal, so is $S$. Note that $S \cap H$ is the intersection of two quadrics. From the exact sequence

$$
0 \rightarrow \mathscr{T}_{s}(1) \rightarrow \mathscr{T}_{s}(2) \rightarrow \mathscr{T}_{S \cap H_{1} H}(2) \rightarrow 0
$$

and Bezout's theorem, one sees that $S$ is the intersection of two quadrics. There are several ways to check that any such $S$ contains a line, contradicting the 2-spannedness. One can work in the Grassmannian $G$ of line in $\mathbf{P}^{4}$, take as $X$ the codimension 3 ([GH, p. 739] cycle of lines contained in a fixed smooth quadric and note that $X^{2} \neq 0$. Or one can use the fact that $S$ is a Del Pezzo surface, obtained by blowing-up 5 suitable points; a line on $S$ is the strict transform on $S$ of the conic through these 5 points ([D] in positive characteristic). Note that this part of the theorem, i.e. the inequality $\operatorname{dim}(W) \geq$ 6 , works even if the base field has positive characteristic.

Now assume $\operatorname{dim}(W)=6$. By a theorem of Le Barz ([LB, p. 182]), the cycle of trisecant lines to $S \cap H$ has degree $t(d, g):=(d-2)(d-3)(d-4) / 6-g(d-4)$; since by assumption $S \cap H$ has a finite number of trisecant lines (indeed none) this number represents the number of trisecant lines to $S \cap H$, counted with suitable multiplicities. Thus $t(d, g)=0$. If $d=4$, then $S$ is a minimal degree 
surface, hence either a Veronese surface or a scroll. Since a scroll contains infinitely many lines, the latter case is impossible. Thus we may assume $d>4$. Since $t(d, g)=0$, we find $g=(d-2)(d-3) / 6$. Let $m_{1}, \varepsilon_{1}, \mu_{1}$, be defined by: $m_{1}:=[(d-1) / 4], \varepsilon_{1}:=d-4 m-1, \mu_{1}:=1$ if $\varepsilon_{1}=3, \mu_{1}:=0$ otherwise. Set $\pi_{1}(d, 4):=m_{1}\left(m_{1}-1\right) / 2+m_{1}\left(\varepsilon_{1}+1\right)+\mu_{1}$. First assume $g>\pi_{1}(d, 4)$. By [ACGH, p. 123], if $d>10 S \cap H$ is contained in a minimal degree surface $T$ of $H, \operatorname{deg}(T)=3 . T$ is either a smooth scroll or the cone over a rational normal curve in $\mathbf{P}^{3}$. First assume that $T$ is a cone. Let $P$ be the vertex of $T$. Since $S \cap H$ has no "trisecant line", it is smooth, and $g>0, S \cap H$ does not contain $P$ and intersects each line of $T$ in two points. The minimal desingularization of $T$ is the Hirzebruch surface $F_{3} . \operatorname{Pic}\left(F_{3}\right)$ has as basis $h$, $f$ ( $f$ is the fiber while $h$ is contracted to $P$ ) with the relations: $h^{2}=-3$, $h \cdot f=1, f^{2}=0$. Furthermore the pull-back of the hyperplane class of $T$ is $h+3 f$. The inverse image of $S \cap H$ in $F_{3}$ must have $2 h+6 f$ as its class. Thus $g=2$, contradicting the classification (up to a case) of all 2-spanned polarized surfaces with sectional genus at most 5 , given in [BS, §5]. Now assume that $T$ is a smooth scroll. One check easily that $T$ is isomorphic to the Hirzebruch surface $F_{1}$, with $h$ and $f$ as basis of $\operatorname{Pic}(T)$, with the relations $h^{2}=-1$, $h \cdot f=1, f^{2}=0$, and with $h+2 f$ as class of the hyperplane section. Since $H \cap S$ has $g>0$ and no "trisecant line", the class of $H \cap S$ is represented by $2 h+b f$ for some integer $b$. Hence $d=b+2$. By the adjunction formula, we find $g=b-2$, contradiction. The case $d=10$ cannot occur, since $g$ is an integer.

Now assume $d=9$, hence $g=7$. Set $m:=[(d-1) / 3], \varepsilon:=d-1-3 m$, $\pi(d, 4):=3 m(m-1) / 2+m \varepsilon$ ([ACGH, p. 116]). Note that $\pi(9,4)=7=g$. By [ACGH, Theorem 2.5(iii) p. 122], $H \cap S$ has infinitely many trisecant lines, contradiction.

Now assume that $g \leq \pi_{1}(d, 4)$. Checking the 4 possible congruence classes of $d \bmod (4)$, one sees that $d \leq 8$, hence $g \leq 5$. Furthermore $d=7$ is excluded since $g$ is an integer. If $(d, g)=(5,1)$ or $(6,2)$, the hyperplane bundle of $H \cap S$ is non-special, hence $S \cap H$ and $S$ are linearly normal. If $d=8, S \cap H$ (hence $S$ ) must be linearly normal both if the hyperplane section is special or not. Thus we may apply the results in [BS, §5]. By [BS, $\S 5]$, to prove Theorem 1 it is sufficient to consider the case of a smooth $S$, $S$ intersection of 3 quadric hypersurfaces in $\mathbf{P}^{5}$. Such a surface $S$ is not 2spanned if and only if it contains a line, since a line $R$ is "trisecant" to $S$ if and only if $R \subset S$ (Bezout's theorem). Let $G:=G(1,5)$ be the Grassmannian of lines in $\mathbf{P}^{5}, \operatorname{dim}(G)=8$, and $X(Q)$ the class of the lines contained in a fixed smooth quadric $Q . X(Q)$ has codimension 3 in $G$ ([GH, p. 739]). For every $g \in \operatorname{Aut}\left(\mathbf{P}^{5}\right)=\operatorname{Aut}(G)$, we have $g(X(Q))=X(g(Q))$. Hence by Bertini's theorem $([\mathrm{K}])$ the general intersection of 3 quadrics contains no line. Using Bertini's theorem one checks easily that there are smooth $S$ containing a line, hence not 2-spanned; for completeness' sake, we give the details. Take 
a line $R$ and let $A$ be the set of quadrics containing $R$, take $Q^{\prime}$ and $Q^{\prime \prime}$ general in $A$ and set $V:=Q^{\prime} \cap Q^{\prime \prime} ; V$ is smooth; since for every $x \in R$, the set $A(x)$ of quadrics $Q \in A$ with $T_{x} Q=T_{x} V$ has codimension 2 in $A$, the general $Q \in A$ is not tangent to $V$ along $R$; by Bertini's theorem for general $Q \in A, Q \cap V$ is smooth. Any such surface $S$ is a $K 3$ surface; any line $R \subset S$ has self-intersection -2 and $x+1$ as Hilbert polynomial; thus any such $S$ contains at most finitely many lines. Counting dimensions, we see that the set of such $S$ containing at least a line has codimension 1 in the set of smooth complete intersection of 3 quadrics.

\section{ACKNOWLEDGMENTS}

This paper was thought and written in the warm atmosphere of Max-Planck Institut (Bonn). This paper is dedicated to Alessandra.

\section{REFERENCES}

[ACGH] E. Arbarello, M. Cornalba, P. Griffiths, J. Harris, Geometry of algebraic curves, vol. I, Springer-Verlag, 1984.

[BS] M. Beltrametti, A. J. Sommese, On $k$-spannedness for projective surfaces, preprint Max-PlanckInstitut (Bonn).

[BFS] M. Beltrametti, P. Francia, A. J. Sommese, On Reider's method and higher embeddings, preprint.

[D] D. Demazure et al., Seminaire sur les singularities des surfaces, Springer Lect. Notes in Math. 777, Springer-Verlag, 1980.

[GH] P. Griffiths, J. Harris, Principles of algebraic geometry, Wiley-Interscience, 1978.

[K] S. L. Kleiman, The transversality of a general translate, Compositio Math. 28 (1974), 287-297.

[LB] P. Le Barz, Formule multi-secants pour les courbes gauche quelconques, in Enumerative geometry and classical algebraic geometry, p. 165-197, Progress in Math. 24, Birkhäuser.

Department of Mathematics, University of Trento, 38050 Povo (TN), Italy 